\title{
EXPLORING THE IMPACT OF ACCESSIBILITY IN MOOC AND OER: A MULTIVOCAL LITERATURE REVIEW
}

Paola Ingavélez-Guerra, Universidad Politécnica Salesiana, Ecuador, Salvador Otón-Tortosa, Universidad de Alcalá, Spain, António Teixeira, Universidade Aberta, Portugal, Vladimir Robles-Bykbaev, Angel Pérez-Muñoz, Universidad Politécnica

Salesiana, Ecuador

\section{Abstract}

This report presents a review of the accessibility models in Learning Resources and MOOCs with the aim of establishing common terms in the research of the EduTech project and other projects associated with virtual accessibility in member HEIs. This study is based on the search and analysis of articles and publications related to the subject following the MLR format. The results showed a lack of applicability and data that support the current situation in Latin America, however, the experiences of European projects and regulations that support their sustainability, establish guidelines that could guide implementation processes in higher education institutions in partner countries

Keywords: Accessibility, MOOC, REA, MLR, virtual environment.

\section{Introduction}

In recent years a rising awareness of the important of promoting inclusiveness in higher education has led to a surge in research and innovation related to the development of accessible learning opportunities across Europe and Latin America.

Establishing the relationships between concepts of terms associated with learning resources, learning objects, multimedia digital resources, is given by the importance of their use in virtual learning environments. The variation and growth of creating and managing learning resources are related to the exponential development of e-learning and virtual education.

Relevant research from Europe and Latin America set guidelines for the creation and management of accessible learning resources. In this study, concepts related to learning resources, virtual courses and MOOCs are analysed from the accessibility approach, based 
on an online search of academic and grey literature relevant for the research questions. The experiences of the partner institutions of the EduTech project have also been taken into consideration. The article review indicates that several accessibility indicators involved in learning resources and MOOCs are related to WCAG compliance, usability, user experience, learning design, quality regulations.

The results of the research have shown that accessibility is transversal to the evaluation of educational resources and is considered within the tools and models to evaluate the methodology of a course and its technical aspects.

\section{Background}

Virtual education is today the most widely used method in the training of most people, and even more so for those who have a disability. The world forum on education 2015 (Incheon Declaration, 2015) in its Education 2030 Declaration establishes that "It is necessary to take advantage of information and communication technologies (ICT) to strengthen education systems, the dissemination of knowledge, access to information, quality learning and more effective service delivery. It is necessary to identify characteristics that allow to strengthen compliance with digital accessibility”.

\section{Open Educational Resource - OER}

In 1994 Hodgins defined the concept of learning objects and received acceptance for the premise of ease of reuse (Rodriguez-Ascaso et al., 2017). Technological advancement and the use of digital resources in the mediation of learning, makes its concept constantly evolve. The definition of (Chiappe Laverde, 2009) as “... digital entity, self-contained and reusable, with a clear educational purpose, made up of at least three editable internal components..." and the constant coincidence in the characteristics of identification, recovery, detectability, reusability, and interoperability; allows you to delimit it but at the same time understand the variability and cultural evolution of its practice in virtual learning environments. It is in this evolutionary path that legal aspects and reuse licenses are established, which gives rise to the OER (Open Educational Resources). The term "open" involves active participation in 5 activities determined by (Wiley \& Hilton, 2019) such as the 5Rs, retain, reuse, review, remix and redistribute.

\section{Massive Open Online Course - MOOC}

The acronym MOOC was coined in 2008 to refer to the course "Connectivity and Connective Knowledge" offered by Stephen Downes, Principal Investigator at the National Research Council of Canada and George Siemens Associate Director of Research and Development at the University of Manitoba (Shah, 2020; Sanchez-Gordon \& Luján-Mora, 2018). The main characteristic of a MOOC responds to an open online course with massive 
student participation. The materials of a MOOC could be protected by copyright $-\mathrm{xMOOC}$ or use and create OERs under a Creative Commons license - cMOOC (Rodriguez-Ascaso et al., 2017). There are several investigations that seek to classify or evaluate MOOCs. Based on learning characteristics, (Teixeira et al., 2019) points out that an iMOOC could highlight its focus on individual responsibility, interaction, interpersonal relationships, innovation and inclusion, or provide a learning experience marked by social interactions and participation considered as SMOOC (see Figure 1).

\section{Relationship between OER and MOOCS}

Based on the contributions of (Rodriguez-Ascaso et al., 2017; Teixeira et al., 2019) the existing relationships between OER and MOOCs are expressed in Figure 1.

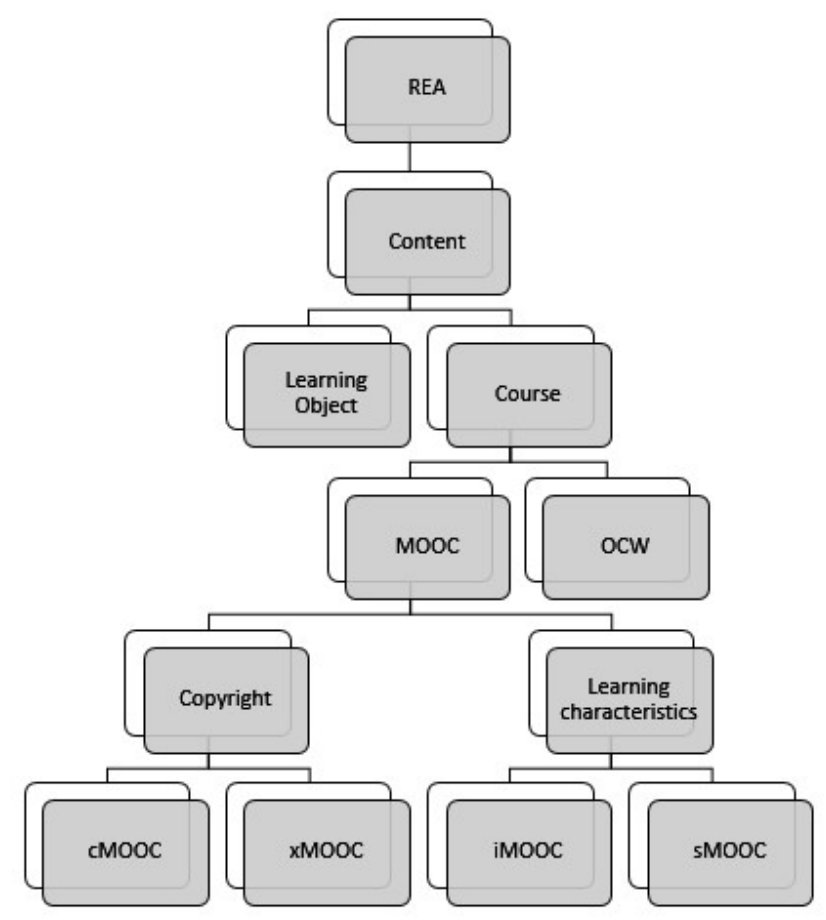

Figure 1. Relation OER \& MOOCs

The existing relationship between OER and MOOCs converges in the identification of their content, whether it is treated individually as a learning object or within a course. Courses can be full, open but copyrighted, usually from an OCW university institution, or open, massive online courses, such as MOOCs. Accessibility is transversal in any of the defined components such as LMS, digital resources, learning objects, virtual learning environments and / or virtual courses and everything that converges in MOOCs and OER.

\section{Accessibility}

Currently, universities face the challenge of providing quality education by strengthening the focus of inclusion and addressing the high rates of exclusion, discrimination, and educational inequality. The creation of conditions for the development of education for all, 
which guarantees quality with equity, implies transformations in the educational system of HEIs, in their cultures, policies, and practices, actively and participatively involving evaluation processes that validate the efforts made. The (ONU, 2006) in the Convention on human rights and its Optional Protocol states in its article 24: "The States Parties recognize the right of persons with disabilities to education. With a view to making this right effective without discrimination and on the basis of equal opportunities, the States Parties shall ensure an inclusive education system at all levels as well as lifelong learning”.

The development of standards establishes rules and requirements that must be met, thus making it possible for the resources to be independent of the platforms, strengthening their interoperability, reuse, durability, updating, scalability, among others. With this, standards are generated for various areas related to learning resources and MOOCs. Teixeira et al. (2013) considers that accessibility in e-learning is not only framed in technology and its interaction, it also requires feedback from the design of learning experiences for all, considering in addition to technology and pedagogy, to ethics.

Methodological proposals focused on the quality of virtual educational resources are based on ISO regulations, establishing guidelines for applying ICTs in teaching, however (Amado-Salvatierra et al., 2018; Sanchez-Gordon \& Luján-Mora, 2018) identify the lack of an accessibility methodology with a holistic and adaptable approach.

Regulations such as (ISO 9241-11, 2018; ISO/IEC 19796-3, 2009; ISO/IEC 24751-3, 2008) establish guidelines that are related to accessibility, however, the applicability and diffusion are still limited. In several countries such as Ecuador, the use of standards from private organizations is not possible until they are considered official standards such as ISO, which is why the WCAG could not be adopted until 2012 when the standard was created (ISO/IEC 40500, 2012) Information technology - W3C Web Content Accessibility Guidelines (WCAG) 2.0.

\section{Methodology}

Studies related to accessibility in OER and their interaction on different platforms have been a considerable effort in addressing the variability of learning that all students present.

It is necessary to carry out a detailed search for accessibility indicators involved in learning resources and MOOCs, and the relationship it maintains with regulations, usability, user experience, learning design, and quality regulations. Accessibility is transversal to the evaluation of educational resources and is considered within the tools and models to evaluate the methodology of a course and its technical aspects.

It is intended to provide a global vision of the current state of initiatives in OER and accessible MOOCs and identify good practices for the creation and management 


\section{Research questions}

The following research questions are asked:

- RQ 1: How is accessible OER created and managed? This question is posed by the diversity of accessibility practices existing in accessible resources. To answer this $\mathrm{RQ}$, relevant previous studies on accessible OER and the educational institutions that influenced their development were analysed.

- RQ 2: Can accessibility be mainstreamed in MOOC courses? The question is posed to establish the phase in which accessibility is considered within the creation of a MOOC course. To respond to this RQ, comparative studies of accessibility of MOOC courses and the establishment of applicable policies or standards in MOOC courses were analyzed.

- RQ 3: What models have been used to promote accessibility in MOOC and OER courses? The question is asked to establish models frequently used when considering accessibility in MOOCs and OER. To answer this RQ, this study investigated the models used in various platforms, identifying best practices, learning outcomes, and degree of satisfaction.

- RQ4: What are the challenges and opportunities that have been addressed in creating and managing accessible OER and MOOCs? The question seeks to establish present and future research trends in the subject of accessibility in OER and MOOC. To respond to this RQ, this study investigates the limitations of existing tools and systems related to accessibility in educational resources. It also summarizes and provides informed recommendations to overcome limitations.

The review of the state of the art on the creation and management of learning resources and accessible MOOCs is a topic that cannot only consider formal literature, the evidence and lack of a measurement consensus requires the inclusion of contextual information, corroborating scientific results with practical experiences. With this, it is necessary to incorporate GL (Grey Literatura) within the structure of the review protocol with the MLR (Multivocal Literature Review) methodology, based on SLR (Systematic Literature Review) (Garousi et al., 2019).

The inclusion criteria in the academic literature respond to being published in the period from January 2013 to December 2019. Writing in English or Spanish. The article must be related to the accessibility strategies in OER and MOOC. The article must respond to relevant research in partner countries or of high connotation in terms of accessibility. Exclusion criteria in the academic literature are those that are not related to accessibility and experiences in virtual education. In google Scholar it is considered the 200 most cited (citation index $>40$ ) 
In grey literature and GOOGLE, after fulfilling the search string, the first 100 searches are considered as inclusion criteria. As exclusion criteria in grey literature, advertisements and commercial images, documents not related to accessibility and experiences in virtual education, broken links or with access to buy books are considered

\section{Quality questions}

In (QA1) the prestige of the author is evaluated where it is detected that all comply with the identification of the author and his expertise. The second criterion (QA2) evaluates the collection of data and procedures that respond to a research methodology and it is concluded that 26 articles present clear objectives and a methodological process supported by limited reliable references in a particular population or situation, 18 present a description of the approach proposed but lacks reliable references or little delimitation of the topic. The third criterion (QA3) examines the objectivity presented, it is concluded that of 35 articles the content of the source is discussed and supported by data while 9 refer to a discussion, however, the opinion is not impartial or is not supported by real data. The fourth criterion (QA4) assesses whether the source presents a clear date of elaboration before which 38 specify a clear date, 5 refer to a period but do not establish a specific date and 1 does not present date of elaboration. The fifth criterion (QA5) assesses whether there is an unpublished and significant contribution to the research, concluding that 28 articles provide innovation and reinforce or current ideas on the subject 13 articles reinforce current ideas, but do not contribute something unique to the research and 3 articles do not contribute innovation or novelty. In the case of GL, the type criterion is added for its quality evaluation and it is concluded that 12 articles are of the 1st level inasmuch as they respond to Books and journals of scientific dissemination and specialized foundations and 1 is considered of $2^{\text {nd }}$ level because it is a presentation. See Figure 2:

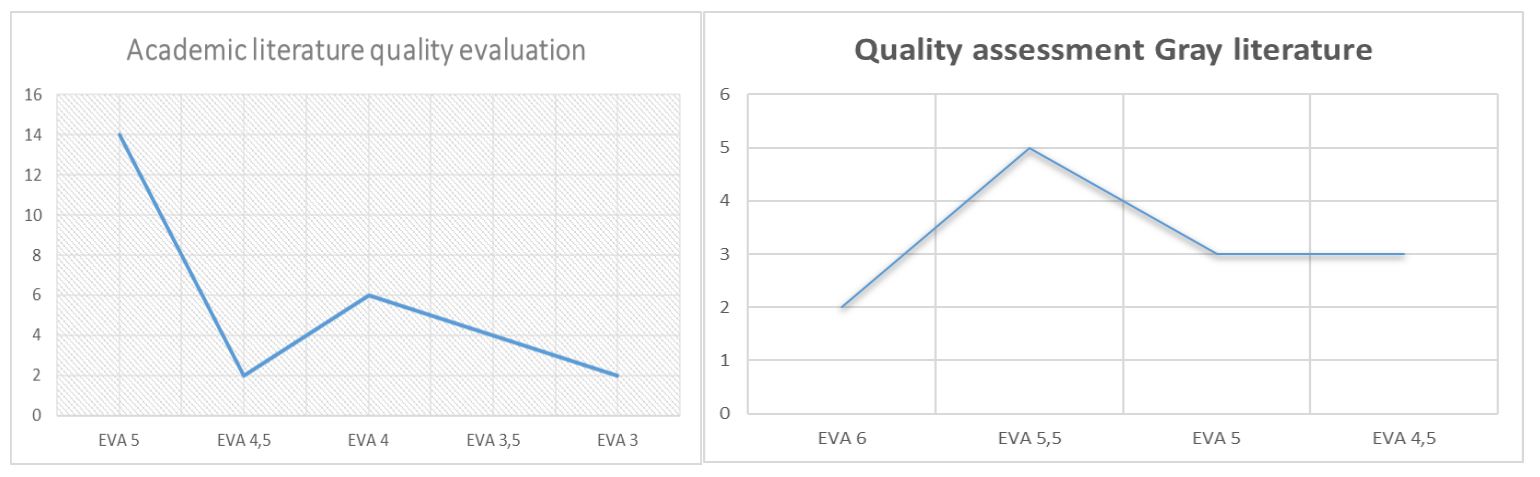

Figure2. Quality assessment

A three-value scale with different descriptions is applied to each question, and the results are used to summarize the quality of the included studies. For this study, the Krippendorff alpha $(\alpha)$ is also calculated for "nominal data, with two observers in order to measure the 
agreement between 2 authors, who carry out the quality assessment independently in a sample of 18 publications.

The data is interpreted in a similar and acceptable way since the alpha value is $74.3 \%$. In case of disagreement, a consensus is reached after discussion with a third author. Finally, a fourth author participates in the supervision of the process and in the establishment of the precision and reliability of the process and the final results.

\section{Analysis of results}

The identification of relevant studies that were selected for this study are included in the analysis for subsequent discussion regarding the RQs.

Of all the studies, $43 \%$ of the selected articles were published in scientific journals, while $26 \%$ belong to highimpact conferences, $29 \%$ are articles and documents on the Internet, which constitute the majority of grey literature. The results of the 44 studies analysed in this research are presented in Annex 1. Each result is presented according to the corresponding research question.

In Figure 3 we can see the distribution of articles according to the year of publication. The interest in research associated with accessibility in MOOC and accessible OER presented between 2013 and 2019.

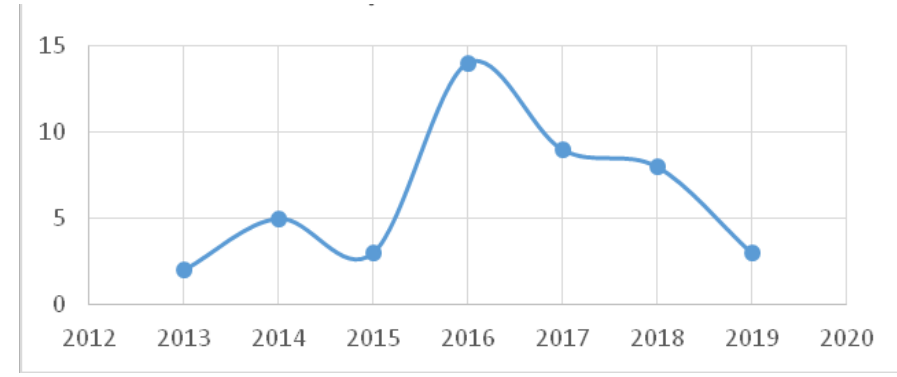

Figure 3. Distribution of studies by year of publication

The volume of publications will increase in 2016, a period in which local laws establish compliance with accessibility regulations, which is why virtual courses and research on the subject take a greater interest.

\section{Conclusion}

As it is based on data from scientific literature and grey literature in a restricted period of time, we consider that the bias was reduced when covering the disciplinary fields of informatics, education, and the search for information and applicability experiences in developed countries and whose legislation endorses accessibility in virtual education. 
There is a shortage of evaluation of accessibility in virtual educational resources and courses, endorsed by people with disabilities. The available studies tend to focus more on design recommendations than on evaluating the effectiveness of their implementation and improvement process. The use of accessibility standards is subjective, in several cases, it responds to evaluative models that, although they consider accessibility as an evaluative metric, it is inconsistent to reach a common implementation process, especially with courses that exceed the legislation of a country. There is a lack of references that establish an important sample of students with disabilities, their follow-up, monitoring, and improvement in the learning design, which requires a longer time to obtain reliability data.

The publication of accessibility information in educational resources, learning objects and MOOCs has a great influence on the effective response of personalized search engines according to the interaction requirements of an educational resource.

Finally, this study reveals that, although the contributions throughout history have generated standards and regulations that have motivated research on the subject, there is a lack of an ideal implementation and frequent use for its application, especially in developing countries. The information from quantitative, qualitative or mixed studies is insufficient to determine the impact on students with disabilities at a general level, so an audit and continuous improvement process is required that involves and commits all the actors within an educational project that supports an accessible MOOC and its learning resources.

\section{References}

Amado-Salvatierra, H. R., González, J. H., \& Tortosa, S. O. (2018). Formalización de un marco metodológico para la implementación de un proyecto educativo virtual accesible. Educación XX1, 21(2). https://doi.org/10.5944/educxx1.15591

Chiappe Laverde, A. (2009). Acerca de lo pedagogico en los objetos de aprendizajereflexiones conceptuales hacia la construccion de su estructura teorica. Estudios Pedagógicos (Valdivia), 35(1), 261-272. https://doi.org/10.4067/S071807052009000100016

Garousi, V., Felderer, M., \& Mäntylä, M. V. (2019). Guidelines for including grey literature and conducting multivocal literature reviews in software engineering. Information and Software Technology, 106, 101- 121. https://doi.org/10.1016/j.infsof.2018.09.006

Incheon Declaration (Vol. 2017). (2015). Retrieved from http://uis.unesco.org/sites/default/files/documents/education-2030incheonframework-for-action-implementation-of-sdg4-2016-en_2.pdf 
Ingavélez-Guerra, P., Otón-Tortosa, S., Teixeira, A., Robles-Bykbaev, V., \& Pérez-Muñoz, A.

Exploring the Impact of Accessibility in MOOC and OER: A Multivocal Literature Review

ISO 9241-11. (2018). ISO 9241-11:2018. Retrieved from

https://www.iso.org/standard/63500.html

ISO/IEC 19796-3. (2009). ISO/IEC 19796-3:2009-Information technology-Learning, education and training-Quality management, assurance and metrics-Part 3:

Reference methods and metrics. ISO. Retrieved from

http://www.iso.org/cms/render/live/en/sites/isoorg/contents/data/standard/04/61/46 159.html

ISO/IEC 24751-3. (2008). ISO/IEC 24751-3:2008-Information technologyIndividualized adaptability and accessibility in e-learning, education and trainingPart 3: "Access for all” digital resource description. ISO. Retrieved from http://www.iso.org/cms/render/live/en/sites/isoorg/contents/data/standard/04/36/43 604.html

ISO/IEC 40500. (2012). ISO/IEC 40500:2012-Information technology-W3C Web Content Accessibility Guidelines (WCAG) 2.0. ISO. Retrieved from http://www.iso.org/cms/render/live/en/sites/isoorg/contents/data/standard/05/86/58 625.html

ONU. (2006). The Secretariat for the Convention on the Rights of Persons with Disabilities (SCRPD) (Vol. 2016). http://www.un.org/disabilities/documents/convention/convoptprot-e.pdf

Rodriguez-Ascaso, A., Boticario, J. G., Finat, C., \& Petrie, H. (2017). Setting accessibility preferences about learning objects within adaptive elearning systems: User experience and organizational aspects. Expert Systems, 34(4), e12187. https://doi.org/10.1111/exsy.12187

Sanchez-Gordon, S., \& Luján-Mora, S. (2018). Research challenges in accessible MOOCs: A systematic literature review 2008-2016. Universal Access in the Information Society, 17(4), 775-789. https://doi.org/10.1007/s10209-017-0531-2

Shah, D. (2020, February 4). Capturing the Hype: Year of the MOOC Timeline Explained. The Report by Class Central [Blog post]. Retrieved from https://www.classcentral.com/report/mooc-hype-year-1/

Teixeira, A., Correia, C. J., Afonso, F., Cabot, A. G., López, E. G., Tortosa, S. O., Piedra, N., Canuti, L., Guzmán, J., \& Sol, M. Á. C. (2013). Inclusive Open Educational Practices: How the Use and Reuse of OER can Support Virtual Higher Education for All. European Journal of Open, Distance and E-Learning, 16(2), Article 2. https://old.eurodl.org/?p=special\&sp=articles\&inum $=5 \&$ abstract $=632 \&$ article $=632$ 
Teixeira, A. M., Mota, J., Pinto, M. do C. T., \& Morgado, L. (2019). Can iMOOCs close the Opportunity Gaps? The contribution of social inclusive pedagogical design. Revista Fuentes, 21(2), 239-252.

Wiley, D., \& Hilton, J. (2019). Definiendo la pedagogía habilitada para REA. Revista Mexicana de Bachillerato a Distancia, 11(21), Article 21. http://dx.doi.org/10.22201/cuaed.20074751e.2019.21.68216

\section{Acknowledgment}

This deliverable has been co-funded by the Erasmus+ Programme of the European Union, project EduTech (609785-EPP-1-2019-1-ES-EPPKA2-CBHE-JP). The European Commission's support for the production of this publication does not constitute an endorsement of the contents, which reflect the views only of the authors, and the Commission cannot be held responsible for any use which may be made of the information contained therein 